Dimensional Analysis and Self-Similarity Methods for Engineers and Scientists 


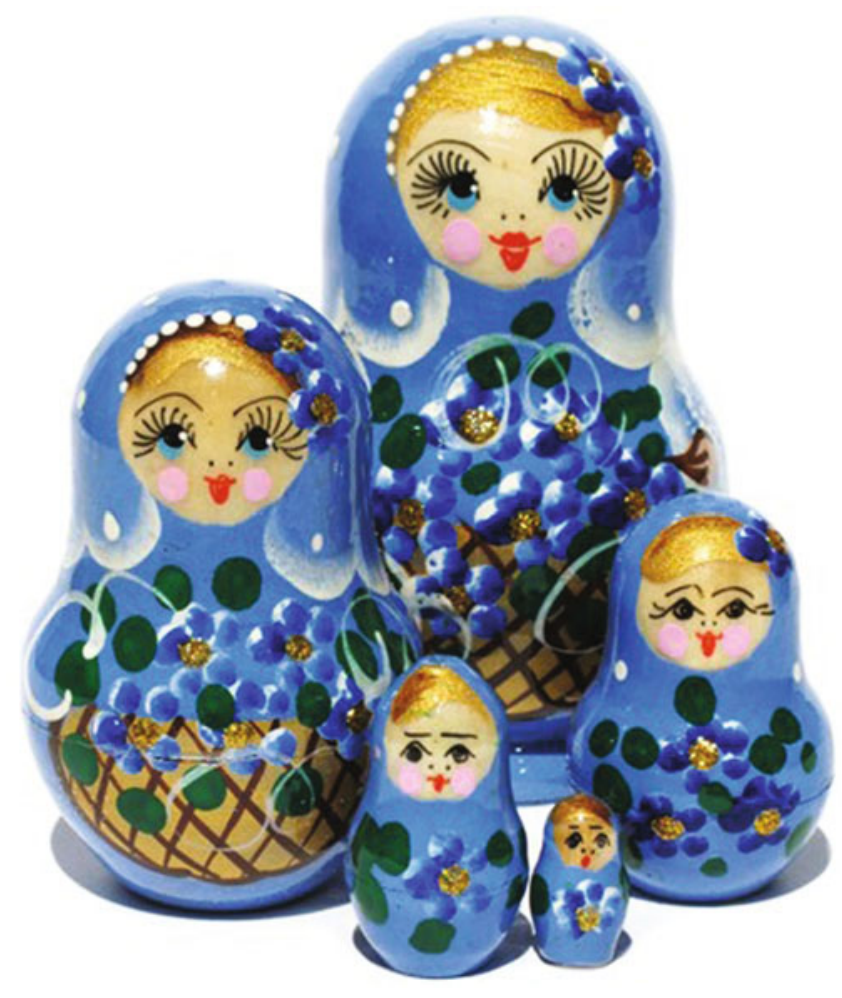


Bahman Zohuri

\section{Dimensional Analysis and Self-Similarity Methods for Engineers and Scientists}

黛 Springer 
Bahman Zohuri

Galaxy Advanced Engineering, Inc.

Albuquerque

NM

USA

ISBN 978-3-319-13475-8

ISBN 978-3-319-13476-5 (eBook)

DOI 10.1007/978-3-319-13476-5

Library of Congress Control Number: 2015932492

\section{Springer Cham Heidelberg New York Dordrecht London}

(C) Springer International Publishing Switzerland 2015

This work is subject to copyright. All rights are reserved by the Publisher, whether the whole or part of the material is concerned, specifically the rights of translation, reprinting, reuse of illustrations, recitation, broadcasting, reproduction on microfilms or in any other physical way, and transmission or information storage and retrieval, electronic adaptation, computer software, or by similar or dissimilar methodology now known or hereafter developed.

The use of general descriptive names, registered names, trademarks, service marks, etc. in this publication does not imply, even in the absence of a specific statement, that such names are exempt from the relevant protective laws and regulations and therefore free for general use.

The publisher, the authors and the editors are safe to assume that the advice and information in this book are believed to be true and accurate at the date of publication. Neither the publisher nor the authors or the editors give a warranty, express or implied, with respect to the material contained herein or for any errors or omissions that may have been made.

Printed on acid-free paper

Springer is part of Springer Science+Business Media (www.springer.com) 
This book is dedicated to my son Sasha, whom I love very much 


\section{Preface}

In physics, engineering, and other aspects of science, dimensional analysis is a tool to find or check relations among physical quantities by using their dimensions. The dimension of a physical quantity is the combination of the basic physical dimensions (usually mass, length, time, electric charge, and temperature) which describe it; for example, speed has the dimension length/time, and may be measured in meters per second, miles per hour, or other units. Dimensional analysis is necessary because a physical law must be independent of the units used to measure the physical variables in order to be general for all cases.

Dimensional analysis is routinely used to check the plausibility of derived equations and computations and to form reasonable hypotheses about complex physical situations. These situations can be tested by experiment or by more developed theories of the phenomena, and to categorize types of physical quantities and units, which are based on their relations. These relations may depend on other units or dimensions if any. Providing a concise and accessible overview of key concepts in dimensional analysis, this book uses cases and examples in engineering and science to show the practical use of dimensional analysis and self-similarity methods in solving complex problems. The text presents all of the mathematical steps along with the main equations. The appendix includes two detailed case studies.

Isaac Newton, who referred to it as the "Great Principle of Similitude," understood the basic principle of dimensional analysis. Nineteenth-century French mathematician Joseph Fourier made important contributions based on the idea that physical laws like $F=M A$ should be independent of the units employed to measure the physical variables. This led to the conclusion that meaningful laws must be homogeneous equations in their various units of measurement, a result that was eventually formalized by Edgar Buckingham with the $\pi$ (Pi) theorem. This theorem describes how every physically meaningful equation involving $n$ variables that can be equivalently rewritten as an equation of $n-m$ dimensionless parameters, where $m$ is the number of fundamental dimensions used. Furthermore, and most importantly, it provides a method for computing these dimensionless parameters from the given variables. 
A dimensional equation can have the dimensions reduced or eliminated through nondimensionalization, which begins with dimensional analysis, and involves scaling quantities by characteristic units of a system or natural units of nature.

The similarity method is one of the standard methods for obtaining exact solutions of Partial Differential Equations (PDEs) in particular non-linear forms. The number of independent variables in a PDE is reduced one-by-one to make use of appropriate combinations of the original independent variables as new independent variables, called "similarity variables."

Solving Boundary Layer problems in Fluid Mechanic and Fluid Dynamics, when encountering non-linear partial differential equation beyond order two, will require usage of Dimensional Analysis and Similarity Method. We have introduced few examples of such problems (i.e. Blasius Equation in Appendix-E). Another example of such usage is in the area of dealing with mathematics and physics of Soliton Wave Partial Differential Equation and their non-linearity behavior. Such issues also can be encountered in physics of laser driven pellet for inertial confinement application and strong shock associated with pellet implosion, where the self-similarity of second kind approach may be used (i.e. Guderley Problem), which is also demonstrated in this book.

In some cases Dimensional Analysis does not provide an adequate approach to establish a solution of a certain eigenvalue problem in nonlinear form which gives rise to the need to discuss similarity method as another approach. In particular, simple cases dealing with reduction of a partial differential equation to an ordinary differential equation in an ordinary way that we have learned in any classical text of same type. In more complex scenarios dealing with boundary-value problem for system of ordinary equations with conditions at different ends of an infinite interval, construction requires a self-similar solution that is more efficient way of solving such complex bounder value problem for the system of ordinary equations directly. In a specific instance, the passage of the solution into a self-similar intermediate asymptotic allows not to have a need to return to the partial differential equations, indeed, in many cases, the self-similarity of intermediate asymptotic can be established and the form of self-similar intermediate asymptotic obtained from dimensional considerations.

The mathematical topics of chaos and fractals are presented both in the body of the book and in the appendices. The chaos and fractals are particularly appropriate in this regard: they are timely-many ideas in these fields were first conceived during the students' lifetimes; they are applicable-fields as diverse as medicine, business, geology, art, and music have adopted ideas from these areas; and they are beautifulthere is something in the gorgeous computer generated images of objects such as the Mandelbrot set, Julia sets, the Koch snowflake, and others that capture students' interest and enthusiasm.

Although the book does not provide any exercises at the end of each chapter, throughout the book numerous examples are provided for the appropriate chapter and sections. Thus, the reader will have ample practical examples of dimensional problems instead of facing a cut and dry abstract approach as existing books of this subject follow. 


\section{Acknowledgments}

I am indebted to the many people who aided me, encouraged me, and supported me beyond my expectations. Some are not around to see the results of their encouragement in the production of this book, yet I hope they know of my deepest appreciations. I especially want to thank Nancy Reis and Dr. Patrick McDaniel of Sandia National Laboratories who both put the idea to me to write this book based on some work that I was contracting with them. Dr. McDaniel, to whom I am deeply indebted, has extensively helped me develop and modify new computer codes and has continuously given his support without hesitation. He has always kept me going in the right direction during the writing of this book.

Thanks also go to Dr. Ali Reza Mokhtari, physics professor at University of Ferdosi, Mashhad, Iran, who has been a true friend since high school and has been there to support me with his comments and reviews of this book for me. To other friends and professors including Stanford University Professor Barnet, and University of California at Berkeley Professor G. I. Barenblatt, who taught the subject of dimensional analysis and who I had the honor of being one of his many students as well as Professor Alan Dorsey of University of Warwick whom helped with some content of Chap. 1. I enjoyed them both while I was learning from them. William Kemp of the Air Force Weapon Laboratory at Albuquerque, New Mexico, has really been a true friend and remains one. Finally, I am indebted to many people and to the individuals and organizations that granted me, permission to reproduce copyrighted materials and published figures.

Above all, I offer very special thanks to my late mother and father, and to my children, in particular, my son Sasha. They have provided constant interest and encouragement, without which this book would not have been written. Their patience with my many absences from home and long hours in front of the computer to prepare the manuscript are especially appreciated. 


\section{Contents}

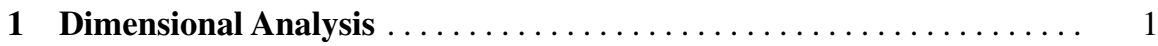

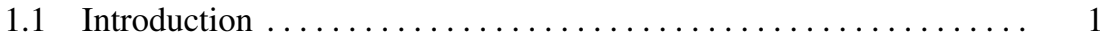

1.2 Dimensional Analysis and Scaling Concept ............... 7

1.2.1 Fractal Dimension........................ 7

1.2.2 Creating Fractals (The Snowflake Curve and Other Fractals) 13

1.2.3 Some Other Fractals .......................... 15

1.2.4 Other Types of Fractals $\ldots \ldots \ldots \ldots \ldots \ldots \ldots \ldots \ldots \ldots \ldots$

1.3 Scaling Analysis and Modeling $\ldots \ldots \ldots \ldots \ldots \ldots \ldots \ldots \ldots \ldots \ldots \ldots$

1.4 Mathematical Basis for Scaling Analysis . .............. 17

1.5 Dimensions, Dimensional Homogeneity, and Independent

Dimensions ................................. 19

1.6 Buckingham's $\pi(\mathrm{Pi})$ Theorem...................... 22

1.6.1 Summarizing the Buckingham's $\pi(\mathrm{Pi})$ Theorem ......... 28

1.6.2 Alternative Mathematical Description of Buckingham's

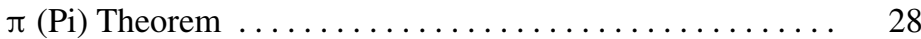

1.6.3 Determination of $\pi(\mathrm{Pi})$ Terms . . . . . . . . . . . . . 29

1.6.4 Some Examples of Buckingham's $\pi(\mathrm{Pi})$ Theorem........ 31

1.7 Oscillations of a Star .............................. 42

1.8 Gravity Waves on Water .......................... 43

1.9 Dimensional Analysis Correlation for Cooking a Turkey ......... 45

1.10 The Problem of an Implosion and Explosion at a Point ........ 51

1.10 .1 Energy in a Nuclear Explosion .................. 52

1.10.2 Energy in a High Intense Implosion . . . . . . . . . . . 65

1.11 Similarity and Estimating $\ldots \ldots \ldots \ldots \ldots \ldots \ldots \ldots \ldots \ldots \ldots \ldots \ldots \ldots \ldots \ldots$

1.12 Self-Similarity ................................. 71

1.13 General Results of Similarity ...................... 83

1.13.1 Principles of Similarity $\ldots \ldots \ldots \ldots \ldots \ldots \ldots \ldots \ldots \ldots \ldots$

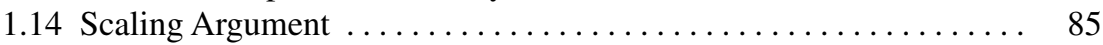

1.15 Similarity and Scaling ............................... 85

1.16 Similarity Transformation $\ldots \ldots \ldots \ldots \ldots \ldots \ldots \ldots \ldots \ldots . \ldots \ldots$

1.17 Self-Similarity Solutions of the First and Second Kind ........ 87

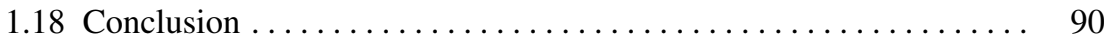

References .................................... 90 
2 Similitude Theory and Applications $\ldots \ldots \ldots \ldots \ldots \ldots \ldots \ldots \ldots, 93$

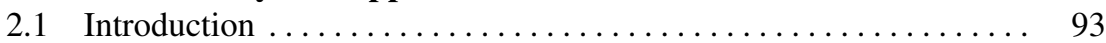

2.1.1 Geometric Similarity $\ldots \ldots \ldots \ldots \ldots \ldots \ldots \ldots \ldots \ldots \ldots \ldots$

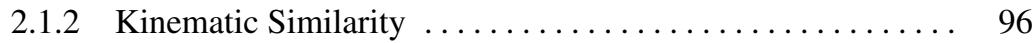

2.1 .3 Dynamic Similarity $\ldots \ldots \ldots \ldots \ldots \ldots \ldots \ldots \ldots \ldots \ldots \ldots$

2.1.4 Scaling Laws ............................... 101

2.1.5 Magnitudes of Forces ....................... 102

2.2 Magnitudes of Different Forces ...................... 103

2.2.1 Inertia Forces............................. 103

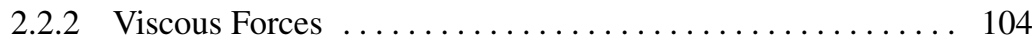

2.2 .3 Pressure Forces ............................... 104

2.2 .4 Gravity Forces ............................. 105

2.2.5 Capillary or Surface Tension Forces .............. 105

2.2.6 Compressibility or Elastic Forces .................. 105

2.3 Dynamic Similarity of Flows governed by Viscous, Pressure and Inertia Forces ............................... 109

2.4 Dynamic Similarity of Flows with Gravity, Pressure and Inertia Forces ............................. 110

2.5 Dynamic Similarity of Flows with Surface Tension

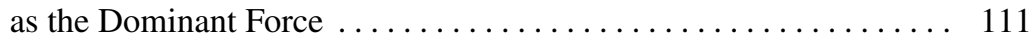

2.6 Dynamic Similarity of Flows with Elastic Force ............. 111

2.7 Dimensional Analysis and Physical Similarity ............. 112

2.7.1 Types of Physical Similarity . . . . . . . . . . . . . . . 113

2.7.2 The Application of Dynamic Similarity . . . . . . . . . . . . 114

2.7.3 Dimensions of Physical Quantities ................. 115

2.7.4 The Buckingham's Pi Theorem ................. 116

2.7.5 Dimensional Analysis of a Problem ............... 116

2.8 Typical Applications . . . . . . . . . . . . . . . . . . . . . . 116

2.9 Dimensional Analysis to Obtain Similarity Parameters.......... 117

2.9.1 Using Buckingham Pi theory to Obtain Similarity Parameters ............................... 117

2.9.2 Model Law $\ldots \ldots \ldots \ldots \ldots \ldots \ldots \ldots \ldots \ldots \ldots \ldots \ldots \ldots$

2.10 Application of Dimensional Analysis and Similarity in Fluid Dynamics ..................................... 122

2.10.1 Dimensional Analysis ....................... 122

2.11 Further Discussion of Buckingham Pi Theorem ........... 123

2.12 Determination of Pi Terms ........................ 123

2.12.1 Method of Repeating Variables .................. 124

2.12.2 Selection of Variables ........................ 131

2.12.3 Determination of Reference Dimensions ............. 132

2.12.4 Uniqueness of Pi Terms ...................... 136

2.13 Determination of Pi Terms by Inspection . . . . . . . . . . . 137

2.14 Common Dimensionless Groups ...................... 139

2.15 Rayleigh's Indicial Method ........................ 144

2.16 The Purposes and Usefulness of Dimensional Analysis .......... 149 
2.17 Development of Prediction Equations . . . . . . . . . . . . . . . 157

2.18 Similarity and Similar System . . . . . . . . . . . . . . 158

2.18.1 Similarity, Modeling, and Estimating . . . . . . . . . . . 159

2.19 Dissimilarity and Dissimilar System . . . . . . . . . . . . . 161

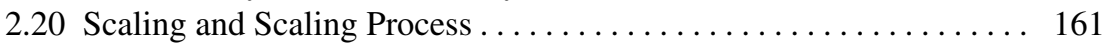

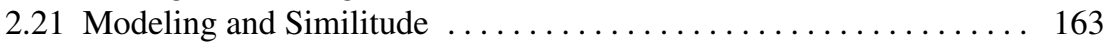

2.21.1 Benefits of Models . . . . . . . . . . . . . . . . . . . . . . 166

2.21.2 Theory of Models ...................... 167

2.21.3 Models and Mathematical Modeling Techniques and Their

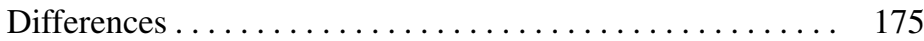

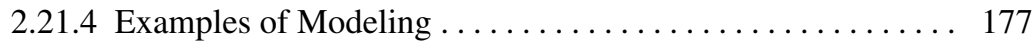

2.21.5 The Modeling Process, Proportionality, and Geometric

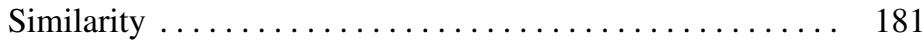

2.21.6 Structural Models . . . . . . . . . . . . . . . . . . . . 183

2.22 Distorted Models . . . . . . . . . . . . . . . . . . . . . . . . . . . . 183

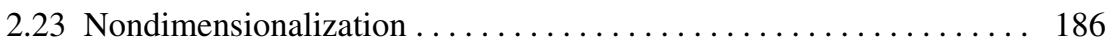

References .................................... 192

3 Dimensional Analysis and Intermediate Asymptotic . . . . . . . . . . 195

3.1 Introduction . . . . . . . . . . . . . . . . . . . . 195

3.2 Similarity Solutions for Partial and Differential Equations . . . . . . . . 198

3.3 Asymptotic Analysis and Singular Perturbation Theory . . . . . . . . . 200

3.4 Regular and Singular Perturbation Problems .............. 201

3.5 Eigenvalue Problems ........................ 201

3.6 Quantum Mechanics ............................ 202

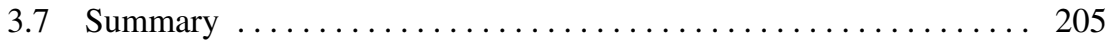

References ............................... 207

4 Similarity Methods for Nonlinear Problems . . . . . . . . . . . . . . . . . 209

4.1 Similarity Solutions for Partial and Differential Equations . . . . . . . . 209

4.2 Fundamental Solutions of the Diffusion Equation Using

Similarity Method ...................... 212

4.3 Similarity Method and Fundamental Solutions of the Fourier Equation ............................ 214

4.4 Fundamental Solutions of the Diffusion Equation; Global Affinity .. 219

4.5 Solution of the Boundary-Layer Equations for Flow Over a Flat Plate ................................ 225

4.6 Solving First Order Partial Differential Equations using Similarity Method ..................... 230

4.6.1 Solving Quasilinear Partial Differential Equations of First Order Using Similarity . . . . . . . . . . . . . . 236

4.6.2 The Boundary Value Problem for a First Order Partial Differential Equation..................... 241

4.6.3 Statement of the Cauchy Problem for First Order Partial Differential Equation. 
4.7 Exact Similarity Solutions on Nonlinear Partial Differential Equations . . . . . . . . . . . . . . . . . . . . . . . . . 245

4.8 Asymptotic Solutions by Balancing Arguments . . . . . . . . . . 247 References . . . . . . . . . . . . . . . . . . . . . . . . . . . . . . . 251

5 Similarity Methods and Dimensional Analysis in Engineering

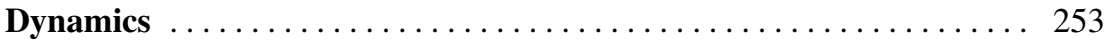

5.1 Introduction to Similarity and Analogy .............. 253

5.2 Infinite Dimensional Analysis . . . . . . . . . . . . . . . . 254

5.2.1 Economy and Infinite Dimensional Analysis . . . . . . . . 255

5.2.2 Quantum Probability and Infinite Dimensional Analysis ... 256

5.2.3 Heat Kernels Measures and Infinite Dimensional Analysis . . 256

5.3 Unsteady Motion of Continuous Media and Self-Similarity Methods 257

5.3.1 Dimensional Analysis and Concept of Self-Similarity

Motion of Medium ...................... 260

5.4 Dimensional Analysis and Physical Similarity of Lossy

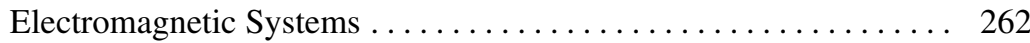

5.5 Extended Self-Similarity in Geophysical and Geological Applications .............................. 263

5.6 Application of Dimensional Analysis and Group Theory

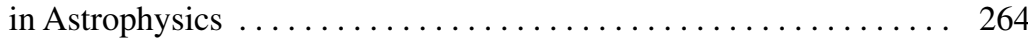

5.7 Visual Similarity Based Three Dimensional Model . . . . . . . . . . . . . . 264

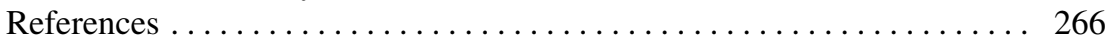

Appendix A: Simple Harmonic Motion . . . . . . . . . . . . . . 269

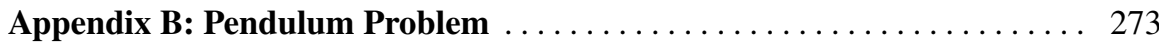

Appendix C: Some Examples of Dimensional Analysis and Similitude . . . 279

Appendix D: Self-Similarity Chaos Game to Sierpinski Triangle . . . . . . 293

Appendix E: Similarity Solutions Methods for Partial Differential

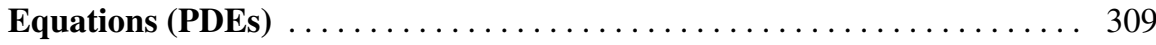

Appendix F: Thermal Explosions . . . . . . . . . . . . . . . . . . . . . . 349

Appendix G: Dimensionless Analysis of Heat Conduction or Diffusion

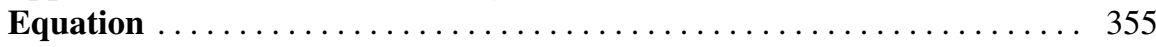

Appendix H: Definition of Some Common Quantities and Their

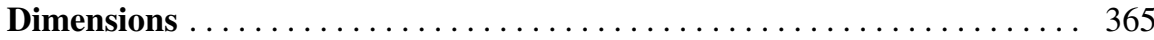

Appendix I: Some Common Liquids Information $\ldots \ldots \ldots \ldots \ldots$. . . . . 367

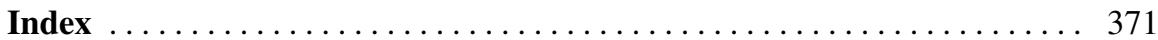




\section{About the Author}

Dr. Bahman Zohuri is currently at Galaxy Advanced Engineering, Inc. a consulting company that he started in 1991, when he left the semiconductor and defense industries after many years working as a chief scientist. After graduating from University of Illinois in field of Physics and Applied Mathematics, he joined Westinghouse Electric Corporation where he performed thermal hydraulic analysis and natural circulation for Inherent Shutdown Heat Removal System (ISHRS) in the core of a Liquid Metal Fast Breeder Reactor (LMFBR) as a secondary fully inherent shut system for secondary loop heat exchange. All these designs were used for Nuclear Safety and Reliability Engineering for Self-Actuated Shutdown System. He designed the Mercury Heat Pipe and Electromagnetic Pumps for Large Pool Concepts of LMFBR for heat rejection purpose for this reactor around 1978 where he received a patent for it. He later on was transferred to defense division of Westinghouse where he was responsible for the dynamic analysis and method of launch and handling of MX missile out of canister. The results are applied to MX launch seal performance and muzzle blast phenomena analysis (i.e. missile vibration and hydrodynamic shock formation). He also was involved in analytical calculation and computation in the study of Nonlinear Ion Wave in Rarefying Plasma. The results are applied to the propagation of "Soliton Wave" and the resulting charge collector traces, in the rarefactions characteristic of the corona of the a laser irradiated target pellet. As part of his graduate research work at Argonne National Laboratory, he performed computation and programming of multi-exchange integral in surface physics and solid state physics. He holds different patent in areas such as diffusion processes and design of diffusion furnace while he was senior process engineer working for different semiconductor industries such as Intel, Varian, and National Semiconductor corporations. Later on he joined Lockheed Missile and Aerospace Corporation as Senior Chief Scientist and was responsible Research and Development (R\&D) and the study of vulnerability, survivability and both radiation and laser hardening of different components Strategic Defense Initiative known as Star Wars. This included of payload (i.e. IR Sensor) for Defense Support Program (DSP), Boost Surveillance and Tracking Satellite (BSTS) and Space Surveillance and Tracking Satellite (SSTS) against laser or nuclear threat. While in there, he also studied and performed the analysis of characteristics of laser beam and nuclear radiation interaction with materials, Transient Radiation Effects in 
Electronics (TREE), Electromagnetic Pulse (EMP), System Generated Electromagnetic Pulse (SGEMP), Single-Event Upset (SEU), Blast and, Thermo-mechanical, hardness assurance, maintenance, device technology.

Under his consulting company, Galaxy Advanced Engineering, Bahman Zohuri worked with Sandia National Laboratories (SNL), where he was supporting development of operational hazard assessments for the Air Force Safety Center (AFSC) in concert with other interested parties. The results are intended for inclusion in Air Force Instructions (AFIs) specifically issued for Directed Energy Weapons (DEW) operational safety. He designed a comprehensive library of detailed laser tools for Airborne Laser (ABL), Advanced Tactical Laser (ATL), Tactical High Energy Laser (THEL), Mobile/Tactical High Energy Laser (M-THEL), etc.

He was also responsible on SDI computer programs involved with Battle Management C3I (Command, Control, Communication and Intelligent) and Artificial Intelligence (AI), and autonomous system. He is author few publications and holds various patents such as Laser Activated Radioactive Decay and Results of ThruBulkhead Initiation.

Recently, Bahman Zohuri has published other books under the following title;

1. Heat Pipe Design and Technology: A Practical Approach

2. Thermodynamics in Nuclear Power Plant Systems

3. Thermal Hydraulic Analysis of Nuclear Reactors

4. Combined Cycle Driven Efficiency of Next Generation Nuclear Power Plants

5. High Energy Laser (HEL) Tomorrow's Weapon in Directed Energy Weapons

Dr. Zohuri has also published other books in various fields of physics and nuclear engineering that can be found on Amazon.com and other retailers. 